\title{
Hybrid Projective Dislocated Synchronization of Liu Chaotic System Based on Parameters Identification
}

\author{
Yanfei Chen \\ College of Science, Guilin University of Technology \\ Guilin 541004, China
}

Tel: 86-139-7838-5785Ｅ-mail: chenyanfei2010@126.com

\author{
Zhen Jia (Corresponding author) \\ College of Science, Guilin University of Technology \\ Guangxi Key Laboratory of Spatial Information and Geomatics, Guilin University of Technology \\ Guilin 541004, China \\ Tel: 86-159-0787-8165Ｅ-mail: jjjzzz0@163.com
}

The research is financed by the National Natural Science Foundation of China (Grand No.61164020) and the Natural Science Foundation of Guangxi, China (No.2011GXNSFA018147).

Received: December 12, $2011 \quad$ Accepted: December 29, $2011 \quad$ Published: February 1, 2012

doi:10.5539/mas.v6n2p16

URL: http://dx.doi.org/10.5539/mas.v6n2p16

\begin{abstract}
This letter discusses hybrid projective dislocated synchronization of Liu chaotic system with five uncertain parameters. Based on adaptive technique, the hybrid projective dislocated synchronization of Liu chaotic system is achieved by designing a novel nonlinear controller. Furthermore, the parameters identification is realized simultaneously. A sufficient condition is given and proved theoretically by Lyapunov stability theory and LaSalle's invariance principle. Finally, the numerical simulations are provided to show the effectiveness and feasibility of the proposed method.
\end{abstract}

Keywords: Hybrid projective dislocated synchronization, Parameters identification, Liu chaotic system, Adaptive technique

\section{Introduction}

Since the pioneering work on chaos synchronization by Pecora and Carroll in 1990 (Pecora, 1990), chaos synchronization has attracted much attraction due to its potential applications in many practical engineering fields, such as secure communication, information processing, chemical reaction, and so on. In the past two decades, many types of synchronization phenomena have been studied, namely, complete synchronization (Lu, 2005), generalized synchronization (Jia, 2008), phase synchronization (Ho, 2002), lag synchronization (Chen, 2007), etc. Meanwhile, many schemes for chaos synchronization have been proposed, including linear and nonlinear feedback approach (Wang, 2006; Jia, 2007), adaptive technique (Jia, 2007), coupled method (Chen, 2011), impulsive control method (Luo, 2008), among many others.

Mainieri and Rehacek considered a type of chaos synchronization, called projective synchronization (Mainieri, 1999), where the corresponding state vectors of drive-response systems could be synchronized up to a constant scaling factor. In Ref. (Hu, 2007), Hu et al. proposed a dislocated synchronization method. In this paper, we investigate the hybrid projective dislocated synchronization and parameters identification of Liu chaotic system. In this scheme, every state variable of drive system synchronizes other mismatched state variables of response system with different scaling factors. Based on the adaptive technique, a novel controller and parameter adaptive laws are designed such that parameters identification is realized, and hybrid projective dislocated synchronization of Liu chaotic system is achieved simultaneously.

This work is organized as follows. The drive and response systems are described and hybrid projective 
dislocated synchronization errors are theoretically analyzed in section 2. In section 3, a general scheme for hybrid projective dislocated synchronization of Liu chaotic system and parameters identification is proved. Section 4 presents some numerical simulations to show the effectiveness of the proposed scheme. Finally, conclusions are shown.

\section{Problem Formulation}

The Liu chaotic system (Liu, 2004) as the drive system is given by

$$
\left\{\begin{array}{l}
\dot{x}_{1}=a\left(x_{2}-x_{1}\right) \\
\dot{x}_{2}=b x_{1}-k x_{1} x_{3} \\
\dot{x}_{3}=-c x_{3}+h x_{1}^{2}
\end{array}\right.
$$

having a chaotic attractor when $a=10, b=40, c=2.5, h=4, k=1$. Here the Lyapunov exponents of system (1) are found to be $L_{1}=1.64328, L_{2}=0, L_{3}=-14.42$. The phase portrait is shown in Figure 1 .

Considering the drive system (1), the response system is controlled Liu chaotic system as following

$$
\left\{\begin{array}{l}
\dot{y}_{1}=a_{s}\left(y_{2}-y_{1}\right)+u_{1} \\
\dot{y}_{2}=b_{s} y_{1}-k_{s} y_{1} y_{3}+u_{2} \\
\dot{y}_{3}=-c_{s} y_{3}+h_{s} y_{1}^{2}+u_{3}
\end{array}\right.
$$

where the system parameters $a_{s}, b_{s}, c_{s}, h_{s}, k_{s}$ of (2) are unknown, $U=\left[u_{1}, u_{2}, u_{3}\right]^{T}$ is the controller which should be designed. Therefore, the goal of parameters identification and hybrid projective dislocated synchronization is to find an appropriate controller $U=\left[u_{1}, u_{2}, u_{3}\right]^{T}$ and parameter adaptive laws of $a_{s}, b_{s}, c_{s}, h_{s}, k_{s}$, such that the synchronization errors

$$
e_{1}=y_{1}-\lambda_{2} x_{2} \rightarrow 0, e_{2}=y_{2}-\lambda_{3} x_{3} \rightarrow 0, e_{3}=y_{3}-\lambda_{1} x_{1} \rightarrow 0 \text { as } t \rightarrow \infty
$$

and the unknown parameters

$$
\lim _{t \rightarrow \infty} a_{s}=a, \lim _{t \rightarrow \infty} b_{s}=b, \lim _{t \rightarrow \infty} c_{s}=c, \lim _{t \rightarrow \infty} h_{s}=h, \lim _{t \rightarrow \infty} k_{s}=k .
$$

where $\lambda_{1}, \lambda_{2}, \lambda_{3}$ are the scaling factors.

Remark 1 When $\lambda_{1} \neq \lambda_{2}$ or $\lambda_{1} \neq \lambda_{3}$, the hybrid projective dislocated synchronization will appear. When $\lambda_{1}=\lambda_{2}=\lambda_{3}$, projective dislocated synchronization will appear. More in general, dislocated synchronization and dislocated anti-synchronization will appear when $\lambda_{i}=1$ and $\lambda_{i}=-1, i=1,2,3$, respectively.

Remark 2 Here are another four types of hybrid projective dislocated synchronization errors

$$
\text { (I ) }\left\{\begin{array}{l}
e_{1}=y_{1}-\lambda_{1} x_{1}, \\
e_{2}=y_{2}-\lambda_{3} x_{3}, \\
e_{3}=y_{3}-\lambda_{2} x_{2} .
\end{array} \text { (II }\right)\left\{\begin{array}{l}
e_{1}=y_{1}-\lambda_{2} x_{2}, \\
e_{2}=y_{2}-\lambda_{1} x_{1}, \\
e_{3}=y_{3}-\lambda_{3} x_{3} .
\end{array} \text { ( III }\right)\left\{\begin{array} { l } 
{ e _ { 1 } = y _ { 1 } - \lambda _ { 3 } x _ { 3 } , } \\
{ e _ { 2 } = y _ { 2 } - \lambda _ { 1 } x _ { 1 } , } \\
{ e _ { 3 } = y _ { 3 } - \lambda _ { 2 } x _ { 2 } . }
\end{array} \text { (IV) } \left\{\begin{array}{l}
e_{1}=y_{1}-\lambda_{3} x_{3}, \\
e_{2}=y_{2}-\lambda_{2} x_{2}, \\
e_{3}=y_{3}-\lambda_{1} x_{1} .
\end{array}\right.\right.
$$

For these cases, the discussions are similar to the method given in this paper.

\section{Hybrid Projective Dislocated Synchronization of Liu Chaotic System}

In this section, based upon the nonlinear adaptive feedback control technique, a systematic design process of parameters identification and hybrid projective dislocated synchronization of Liu chaotic system under the situation of response system with unknown parameters is provided.

According to the systems (1) and (2), the errors dynamical system can be obtained as follows.

$$
\left\{\begin{array}{l}
\dot{e}_{1}=-a e_{1}-\left(a_{s}-a\right) y_{1}+a_{s} y_{2}+k \lambda_{2} x_{1} x_{3}-b \lambda_{2} x_{1}-a \lambda_{2} x_{2}+u_{1}, \\
\dot{e}_{2}=-c e_{2}+\left(b_{s}-b\right) y_{1}-\left(k_{s}-k\right) y_{1} y_{3}-k y_{1} y_{3}+b y_{1}+c y_{2}-h \lambda_{3} x_{1}^{2}+u_{2}, \\
\dot{e}_{3}=-a e_{3}-\left(c_{s}-c\right) y_{3}+\left(h_{s}-h\right) y_{1}^{2}+h y_{1}^{2}-(c-a) y_{3}-a \lambda_{1} x_{2}+u_{3} .
\end{array}\right.
$$

Obviously, hybrid projective dislocated synchronization of systems (1) and (2) appears if the errors dynamical system (5) has an asymptotically stable equilibrium point $e=0$, where $e=\left[e_{1}, e_{2}, e_{3}\right]^{t}$. 
Thus, we design the controller and parameter adaptive laws as the following theorem.

Theorem Assuming that the Liu chaotic system (1) drives the controlled Liu chaotic system (2), take

$$
\left\{\begin{array}{l}
u_{1}=-a_{s} y_{2}-k \lambda_{2} x_{1} x_{3}+b \lambda_{2} x_{1}+a \lambda_{2} x_{2}, \\
u_{2}=k y_{1} y_{3}-b y_{1}-c y_{2}+h \lambda_{3} x_{1}^{2}, \\
u_{3}=-h y_{1}^{2}+(c-a) y_{3}+a \lambda_{1} x_{2},
\end{array}\right.
$$

and parameter adaptive laws

$$
\left\{\begin{array}{l}
\dot{a}_{s}=y_{1} e_{1}, \\
\dot{b}_{s}=-y_{1} e_{2}, \\
\dot{c}_{s}=y_{3} e_{3}, \\
\dot{h}_{s}=-y_{1}^{2} e_{3}, \\
\dot{k}_{s}=y_{1} y_{3} e_{2} .
\end{array}\right.
$$

Systems (1) and (2) can realize hybrid projective dislocated synchronization and the unknown parameters will be identified, i.e., Eqs. (3) and (4) will be achieved.

Proof Eq. (5) can be converted to the following form under the controller (6)

$$
\left\{\begin{array}{l}
\dot{e}_{1}=-a e_{1}-\left(a_{s}-a\right) y_{1}, \\
\dot{e}_{2}=-c e_{2}+\left(b_{s}-b\right) y_{1}-\left(k_{s}-k\right) y_{1} y_{3}, \\
\dot{e}_{3}=-a e_{3}-\left(c_{s}-c\right) y_{3}+\left(h_{s}-h\right) y_{1}^{2} .
\end{array}\right.
$$

Consider the following Lyapunov function

$$
V=\frac{1}{2}\left[e_{1}^{2}+e_{2}^{2}+e_{3}^{2}+\left(a_{s}-a\right)^{2}+\left(b_{s}-b\right)^{2}+\left(c_{s}-c\right)^{2}+\left(h_{s}-h\right)^{2}+\left(k_{s}-k\right)^{2}\right],
$$

Obviously, $V$ is a positive definite function. Taking its time derivative along with the trajectories of Eqs. (8) and (7) leads to

$$
\begin{aligned}
\dot{V} & =e_{1} \dot{e}_{1}+e_{2} \dot{e}_{2}+e_{3} \dot{e}_{3}+\left(a_{s}-a\right) \dot{a}_{s}+\left(b_{s}-b\right) \dot{b}_{s}+\left(c_{s}-c\right) \dot{c}_{s}+\left(k_{s}-k\right) \dot{k}_{s}+\left(h_{s}-h\right) h_{s} \\
& =-a e_{1}^{2}-c e_{2}^{2}-a e_{3}^{2}=-e^{T} P e \leq 0,
\end{aligned}
$$

It is obvious that $\dot{V}=0$ if and only if $e_{i}=0, i=1,2,3$, namely the set $M=\left\{e=0, a_{s}=a, b_{s}=b, c_{s}=c, h_{s}=h, k_{s}=k\right\}$ is the largest invariant set contained in $E=\{\dot{V}=0\}$ for Eq. (8). So according to the LaSalle's invariance principle (Lasalle, 1960), starting with arbitrary initial values of Eq. (8), the trajectory converges asymptotically to the set $M$, i.e., $e \rightarrow 0, a_{s} \rightarrow a, b_{s} \rightarrow b, c_{s} \rightarrow c, h_{s} \rightarrow h$ and $k_{s} \rightarrow k$ as $t \rightarrow \infty$. This indicates that the hybrid projective dislocated synchronization of Liu chaotic system is achieved and the unknown parameters $a_{s}, b_{s}, c_{s}, h_{s}, k_{s}$ can be successfully identified by using controller (6) and parameter adaptive laws (7). Now the proof is completed.

Remark 3 Taking our adaptive synchronization method, we can not only achieve synchronization but also identify the system parameters.

\section{Numerical Simulation}

In this section, some numerical simulations about the hybrid projective dislocated synchronization and parameters identification between systems (1) and (2) are given to verify the effectiveness and feasibility of the proposed technique. In the numerical simulations, all the differential equations are solved by using the fourth-order Runge-Kutta method.

We assume that the drive signals are from the Liu chaotic system (1) with system parameters $a=10, b=40, c=2,5, h=4, k=1$ and the initial values $x(0)=[2.2,2.4,38]^{T}$, the initial values of controlled Liu chaotic system (2) is $y(0)=[9,-2,-15]^{T}$ and the unknown parameters have zero initial condition. The three scaling factors are chosen as $\lambda_{1}=-2, \lambda_{2}=1, \lambda_{3}=2.8$. The simulation results are shown in Figures 2 and 3. Figure 2 (a)-(c) display the errors state response of systems (1) and (2), Figure 3 shows the identification results of unknown parameters $a_{s}, b_{s}, c_{s}, h_{s}, k_{s}$. 


\section{Conclusion}

In this letter, we introduce an adaptive hybrid projective dislocated synchronization and parameters identification scheme for the Liu chaotic system with the response system parameters unknown. With this scheme we can achieve hybrid projective dislocated synchronization and parameters identification simultaneously. Theoretical proof and numerical simulations demonstrate the effectiveness of the proposed scheme.

It should be pointed out that, although this process is focused on the Liu chaotic system, the systematic design process could be used for many other complex dynamical systems with unknown parameters.

\section{References}

Chen, Y., Chen, X., \& Chen, S. (2007). Lag synchronization of structurally nonequivalent chaotic systems with time delays. Nonlinear Analysis, 66, 1929-1937. http://dx.doi.org/10.1016/j.na.2006.02.033

Chen, J., Lu, J., \& Wu, X. (2011). Bidirectionally coupled synchronization of the generalized Lorenz system. $J$ Syst Sci Complex, 24, 443-448. http://dx.doi.org/10.1007/s11424-010-8323-2

Ho, M. C., Hung, Y. C., \& Chou, C. H. (2002). Phase and anti-phase synchronization of two chaotic systems by using active control. Phys. Lett. A., 296, 43-48. http://dx.doi.org/10.1016/S0375-9601(02)00074-9

Hu, M., \& Xu, Z. (2007). Nonlinear feedback mismatch synchronization of Lorenz chaotic sysytems. Syst Eng Electr, 29(8), 1346-1348.

Jia, Z. (2008). Linear generalized synchronization of chaotic systems with uncertain parameters. $J$ Syst Eng Electr, 19(4), 779-784. http://dx.doi.org/10.1016/S1004-4132(08)60153-X

Jia, Z., Lu, J. A., \& Deng, G. M. (2007). Nonlinear state feedback and adaptive synchronization of hyperchaotic Lü systems. Syst Eng Electr, 29(4), 598-600.

Lasalle, J. P. (1960). The extent of asymptotic stability. Proc. Natl. Acad. Sci. U.S.A., 46(3), 363-365. http://dx.doi.org/10.1073/pnas.46.3.363

Liu, C., Liu, T., Liu, L., \& Liu, K. (2004). A new chaotic attractor. Chaos, Solitons \& Fractals, 22, 1031-1038. http://dx.doi.org/10.1016/j.chaos.2004.02.060

Luo, R. Z. (2008). Impulsive control and synchronization of a new chaotic system. Phys .Lett. A, 372, 648-653. http://dx.doi.org/10.1016/j.physleta.2007.08.010

Lu, J., \& Cao, J. (2005). Adaptive complete synchronization of two identical or different chaotic (hyperchaotic) systems with fully unknown parameters. Chaos, 15(4), 043901. http://dx.doi.org/10.1063/1.2089207

Mainieri, R., \& Rehacek, J. (1999). Projective synchronization in three-dimensional chaotic systems. Phys. Rev. Lett., 82(15), 3042-3045. http://dx.doi.org/10.1103/PhysRevLett.82.3042

Pecora, L. M., \& Carroll, T. L. (1990). Synchronization in chaotic systems. Phys. Rev. Lett., 64(8), 821-824. http://dx.doi.org/10.1103/PhysRevLett.64.821

Wang, F., \& Liu, C. (2006). A new criterion for chaos and hyperchaos synchronization using linear feedback control. Phys.Lett.A, 360, 274-278. http://dx.doi.org/10.1016/j.physleta.2006.08.037

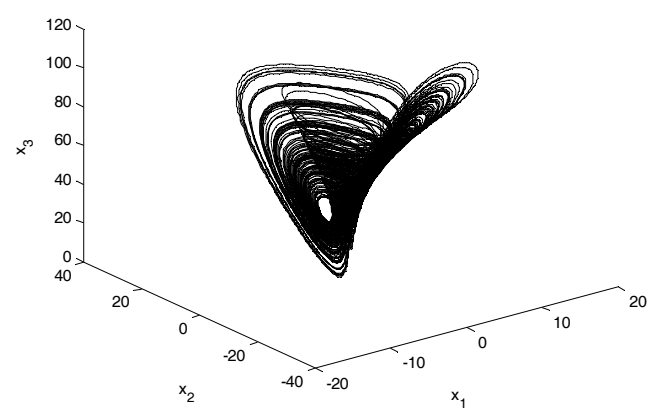

Figure 1. The phase portrait of Liu chaotic system (1) with parameter values

$$
a=10, b=40, c=2.5, h=4, k=1
$$




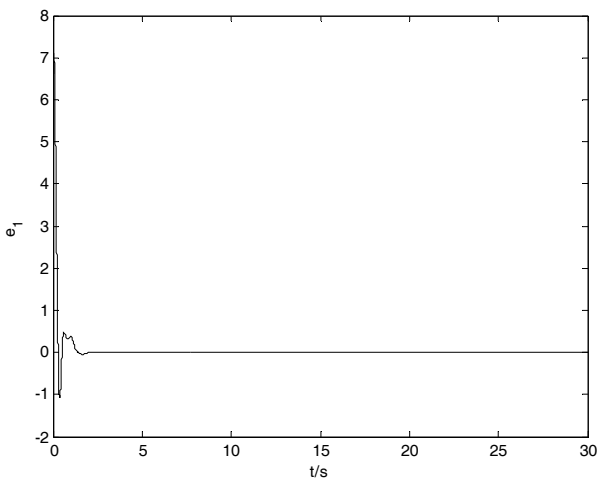

(a)

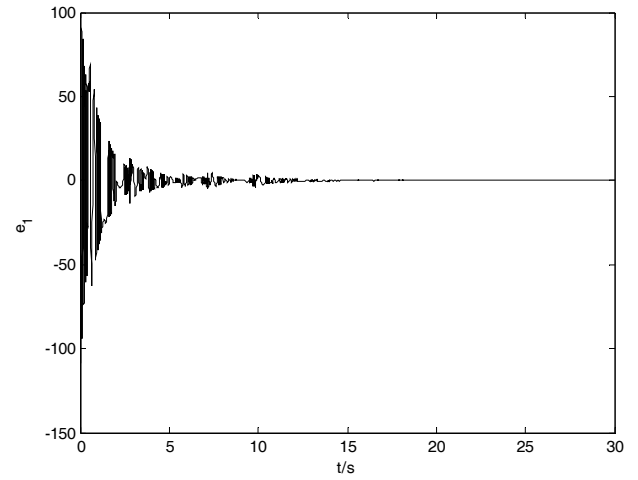

(b)

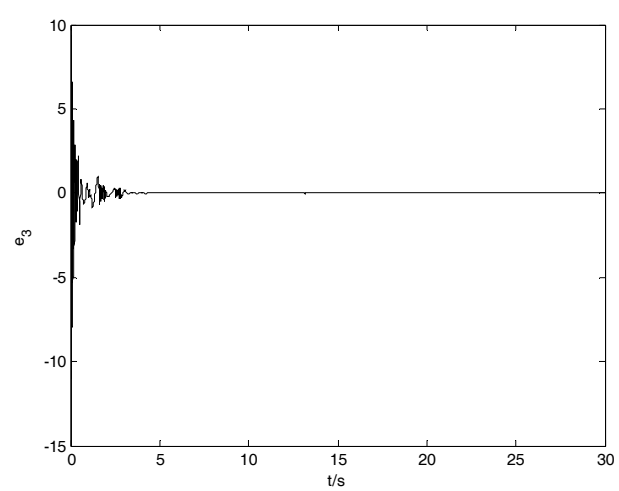

(c)

Figure 2. The synchronization error evolutions of systems (1) and (2):

(a) $e_{1}=y_{1}-x_{2}$; (b) $e_{2}=y_{2}-2.8 x_{3}$; (c) $e_{3}=y_{3}+2 x_{1}$ 


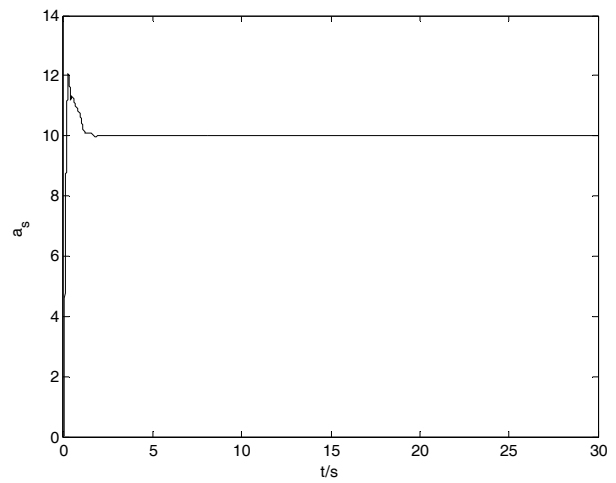

(a)

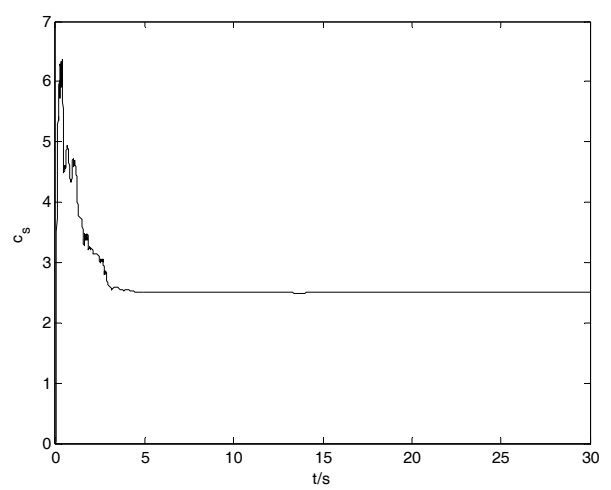

(c)

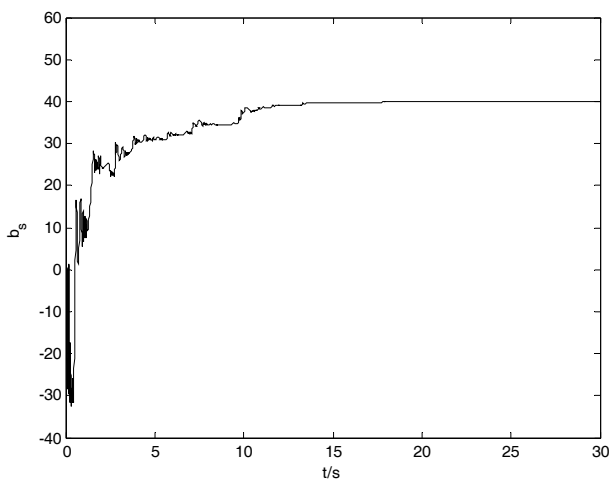

(b)

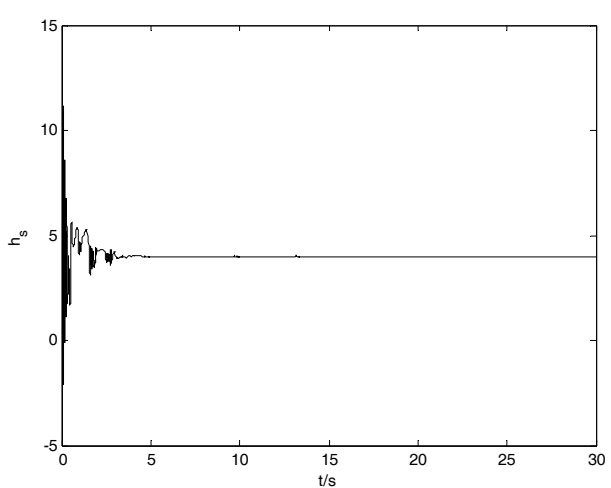

(d)

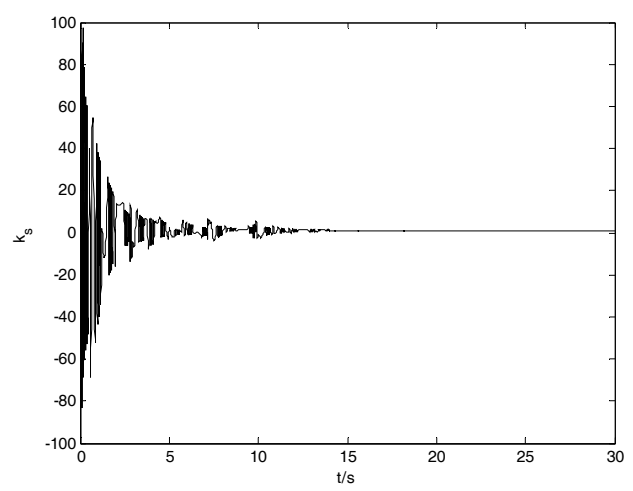

(e)

Figure 3. The parameters identification results of response system (2):

(a) $a_{s}=10$; (b) $b_{s}=40$; (c) $c_{s}=2.5$; (d) $h_{s}=4$; (e) $k_{s}=1$ 